TRANSACTIONS OF THE

AMERICAN MATHEMATICAL SOCIETY

Volume 354, Number 5 , Pages 1947-1959

S 0002-9947(02)02942-2

Article electronically published on January 7, 2002

\title{
ON THE MAXIMAL BOCHNER-RIESZ CONJECTURE IN THE PLANE FOR $p<2$
}

\author{
TERENCE TAO
}

\begin{abstract}
We give a new estimate on the maximal Bochner-Riesz operator in the plane, for $p<2$; as a corollary we obtain an almost everywhere convergence result for certain Bochner-Riesz means. This work was inspired by discussions with Michael Christ and Chris Sogge.
\end{abstract}

\section{INTRODUCTION}

In this paper we shall always be working in the plane $\mathbf{R}^{2}$.

For all $\delta, t>0$, the Bochner-Riesz multipliers $S_{t}^{\delta}$ are defined as

$$
S_{t}^{\delta}=\left(1-\frac{|D|^{2}}{t^{2}}\right)_{+}^{\delta},
$$

where $m(D)$ denotes the multiplier operator

$$
\widehat{m(D) f} f(\xi)=m(\xi) \hat{f}(\xi) \text {. }
$$

We consider the question of finding the pairs $(\delta, p)$ for which $S_{t}^{\delta} f$ converges to $f$ a.e. as $t \rightarrow \infty$, for arbitrary $L^{p}$ functions $f$. For $p>2$ this question was settled by Carbery [6] (see also [8]), who showed that this is the case if and only if $\delta>0, \frac{1}{2}-\frac{2}{p}$.

In the remainder of the paper we consider the more difficult case $1<p<2$; this was posed as an open problem 1 in [22]. By Stein's maximal principle [21] the problem is equivalent to proving the weak-type maximal operator estimate

$$
\left\|\sup _{t>0}\left|S_{t}^{\delta} f\right|\right\|_{p, \infty} \lesssim\|f\|_{p}
$$

for test functions $f$. In 20 this was shown for $\delta>\frac{1}{p}-\frac{1}{2}$. In the converse direction, an old example of Herz shows that (1) fails unless $\delta \geq \frac{2}{p}-\frac{3}{2}$, while the construction of Fefferman [13] shows that (1) fails unless $\delta>0$. In [27] the condition of Herz was strengthened to

Proposition 1.1. 27] The estimate (1) fails unless $\delta \geq \frac{3}{2 p}-1$.

This is proven by considering the counterexample

$$
f\left(y_{1}, y_{2}\right)=e^{2 \pi i \lambda y_{1}} \psi\left(\lambda^{1 / 2} y_{1}, \lambda y_{2}\right)
$$

for some bump function $\psi$ and a parameter $\lambda \gg 1$, and noting that $\left|S_{\lambda|x| / x_{1}} f(x)\right| \sim$ $\lambda^{-1-\delta}$ when $x_{1} \sim|x| \sim 1$. (See [27] for details.)

Received by the editors January 30, 1998.

2000 Mathematics Subject Classification. Primary $42 \mathrm{~B} 15$.

${ }^{1}$ For the cases $p \leq 1$, see e.g. [25, [17, [10]; the case $p=2, \delta=0$ is an extremely difficult open problem, being a higher-dimensional version of the Carleson-Hunt theorem.

(c)2002 American Mathematical Society 


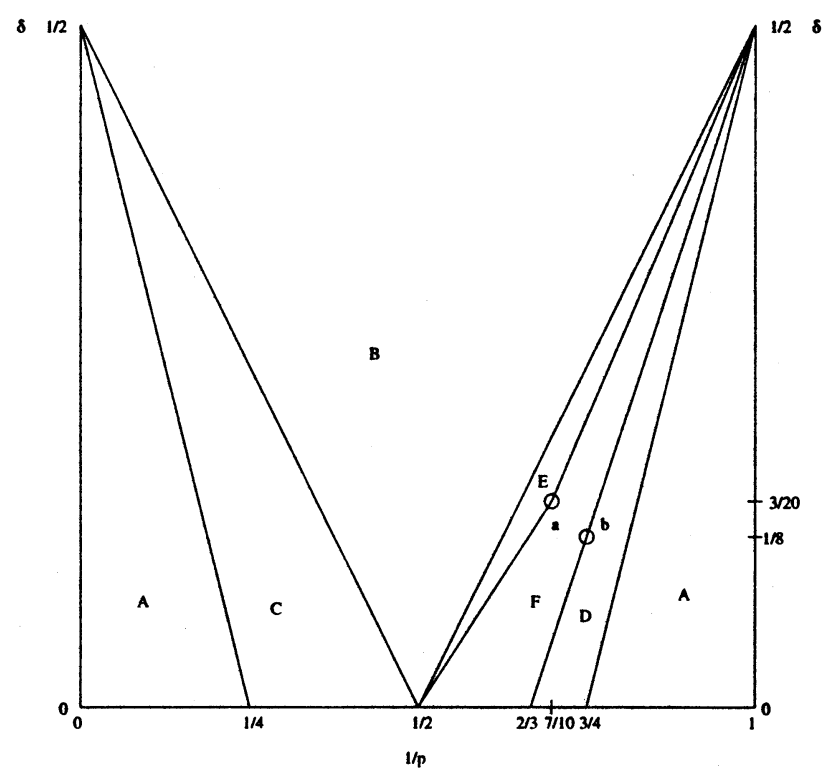

FiguRE 1. A.e. convergence of Bochner-Riesz means in the plane.

The purpose of this paper is to improve upon the positive results in [20]. More precisely, we show

Theorem 1.2. Suppose that $1<p<2$ and

$$
\delta>\max \left(\frac{3}{4 p}-\frac{3}{8}, \frac{7}{6 p}-\frac{2}{3}\right) .
$$

Then (1) holds, and one has almost-everywhere convergence of the Bochner-Riesz means $S_{t}^{\delta} f(x)$ for all $f \in L^{p}$.

We contrast this with the known results in Figure 1 The results of Herz and Stein show that a.e. convergence of Bochner-Riesz means of order $\delta$ for $L^{p}$ functions fails in the regions $A$, but hold in the region $B$. The result of Carbery [6] extends the positive results to $C$, while the counterexample in [27] extends the negative results to $D$. Theorem 1.2 states that the positive results also hold in the region $E$, while the region $F$ remains open, apart from some endpoints. We remark that the situation is simpler and well-understood for radial functions, or for lacunary sequences of Bochner-Riesz means; see [15, 16, [7].

To prove Theorem 1.2, it suffices by interpolation with the classical results to prove (3) for $p=\frac{10}{7}, \delta=\frac{3}{20}+\varepsilon$, where $\varepsilon>0$ is arbitrary. (This corresponds to the point $a$ in Figure 1.) From the reductions 2 in [27 we may localize in space and frequency (giving up an $\varepsilon$ in the $\delta$ index) and reduce ourselves to proving the following estimate.

\footnotetext{
${ }^{2}$ We sketch the derivation of this reduction in an Appendix.
} 
Proposition 1.3. For all $\lambda \gg 1$ we have

$$
\|S f\|_{L_{x}^{q} L_{t}^{\infty}} \leq C_{\varepsilon} \lambda^{-\frac{1}{2}+\delta+\varepsilon}\|f\|_{p}
$$

for $p=q=\frac{10}{7}, \delta=\frac{3}{20}$, and any $\varepsilon>0$. where

$$
S f(x, t)=\int e^{2 \pi i \lambda t|x-y|} a(x, y, t) f(y) d y
$$

and $a(x, y, t)$ is any smooth function supported on the region $t,|x-y| \sim 1,|x|,|y| \lesssim$ 1.

In fact we will be able to improve the $q$ exponent to $q=2$.

Our approach is very much in the spirit of the work of Bourgain and later authors (see e.g. [4], [2, [3], [18, [19, [5]), and employs two basic ideas from that body of work. The first 4 is the introduction of an intermediate scale of $\lambda^{-1 / 2}$ in addition to the natural scales of 1 and $\lambda^{-1}$ that appear in (3). By performing a Taylor approximation of the phase function in a rigorous manner, one can microlocalize the operator $S$, resolving the phase into several distinct pieces. This factors the operator into a "local" part, in which the phase function is linear, and a "global" part, which is handled by Kakeya-type estimates, together with some $L^{2}$ theory to remove the oscillations. For technical reasons we will employ the continuous microlocalization procedure in [4] rather than the discrete decomposition of physical space into $\lambda^{-1 / 2}$-squares which appears elsewhere (e.g. [1]).

Secondly, we will adopt the explicit real interpolation philosophy utilized in e.g. [2], [18], [3], 28], [5]. This means that whenever we obtain a new estimate on the desired operator, we split the function into the portion which can be wellcontrolled by that estimate, plus a remainder. We continue in this manner until all portions of the function have been controlled; the precise way of dividing up the function will depend on parameters to be optimized later. A typical application of this approach is to obtain a good estimate for functions supported on a small square, and then use a Calderón-Zygmund decomposition to break a function into a small number of such functions, together with a remainder which is spread over a sparse set, which will be eventually controlled by another estimate which takes advantage of this sparseness. Although this approach is not as elegant as the usage of standard interpolation theorems, it is more flexible and allows one to reconcile very distinct-looking estimates.

To flesh out the approach outlined in the previous two paragraphs, we will use a number of standard harmonic analysis results, most notably the $L^{4}$ adjoint restriction theorem, a version of Cordoba's $L^{2}$ estimate for Kakeya-type operators, and the standard $L^{2}$ theory for the Fourier transform. The results are not optimal, and one could probably improve upon them by adding better estimates and techniques to the ones listed above.

This work was conducted at MSRI (NSF grant DMS-9701955), and was initiated by some long and fruitful discussions with Chris Sogge and Michael Christ. The author also thanks Tony Carbery for many helpful comments.

\footnotetext{
${ }^{3}$ Our mixed space norms will always be evaluated from right to left; for instance, we have

$$
\|f\|_{L_{x}^{q} L_{t}^{r}}=\left(\int\|f(x, \cdot)\|_{L_{t}^{r}}^{q} d x\right)^{1 / q} .
$$

${ }^{4}$ The author is indebted to Michael Christ for suggesting this line of approach.
} 


\section{Proof of the main estimate}

In the rest of the paper we shall take $\lambda \gg 1$ to be fixed, and suppress all factors which are $O\left(\lambda^{\varepsilon}\right)$ for all $\varepsilon>0$. We will always consider the variables $x, y, t, u, v, r, \omega$ to be constrained so that $|x-y|, t, r \sim 1,|x|,|y|,|u|,|v| \lesssim 1, \omega \in S^{1}$. Any expression of the form $a(\ldots)$ denotes an arbitrary smooth function such that the support of the variables obeys the above constraints; the exact value of $a$ may vary from line to line, but we can justify such changes in amplitude by the stability lemma in an Appendix. We will pretend that the Fourier transforms of such amplitude functions are supported in the ball $B\left(0, \lambda^{\varepsilon}\right)$, since the portion of such functions outside this ball is rapidly decreasing in $\lambda$. Primed variables $\left(x^{\prime}, r^{\prime}\right.$, etc. $)$ will be considered to be constrained in the same manner as their unsubscripted counterparts. Finally, we use $x_{1}, x_{2}$ to denote the $e_{1}$ and $e_{2}$ components of $x$, and similarly for all other variables in $\mathbf{R}^{2}$.

We will say that a quantity $A$ has polynomial size if $\lambda^{-C} \lesssim|A| \lesssim \lambda^{C}$ for some constant $C$. Most quantities we will consider can be assumed to be of polynomial size without loss of generality.

We wish to prove (3) for $p=\frac{10}{7}, q=2, \delta=\frac{3}{20}$, and any $\varepsilon>0$. There are two classical estimates of the form (3) when $1 \leq p \leq 2$, namely

$$
\|S f\|_{L_{x}^{\infty} L_{t}^{\infty}} \lesssim\|f\|_{1}
$$

and 5

$$
\|S f\|_{L_{x}^{2} L_{t}^{\infty}} \lesssim \lambda^{-\frac{1}{2}}\|f\|_{2} .
$$

The results of [20] can be obtained from interpolating between these two estimates.

The Knapp example

$$
f\left(y_{1}, y_{2}\right)=e^{2 \pi i \lambda y_{1}} \psi\left(y_{1}, \lambda^{1 / 2} y_{2}\right),
$$

for a suitable bump function $\psi$, is such that $|S f(x)| \sim \lambda^{-1 / 2}$ for $x_{1} \sim 1,\left|x_{2}\right| \lesssim$ $\lambda^{-1 / 2}$, and therefore shows that the two classical estimates above are sharp. However, the fact that (2) is not a sharp counterexample to (4) suggests that (41) can be sharpened if $f$ is concentrated in a small square. Indeed, we have

Proposition 2.1. If $\psi_{Q}$ is an $L^{\infty}$-normalized bump function adapted to a square $Q$, then

$$
\left\|S\left(\psi_{Q} f\right)\right\|_{L_{x}^{2} L_{t}^{\infty}} \lesssim \lambda^{-1 / 2}|Q|^{1 / 4}\|f\|_{2} .
$$

Proof. W.l.o.g. we may restrict the $y$ variable to the ball $B\left(-2 e_{1}, 1\right)$ and the $x$ variable to the ball $B(0,1)$. We may of course assume that $|Q| \lesssim 1$. We may freeze the $x_{1}$ coordinate, so that we need only prove

$$
\left\|S\left(\psi_{Q} f\right)\right\|_{L_{x_{2}}^{2} L_{t}^{\infty}} \lesssim \lambda^{-1 / 2}|Q|^{1 / 4}\|f\|_{2}
$$

uniformly in $x_{1}$. Henceforth $x_{1}$ is considered fixed.

By the $T T^{*}$ method this is equivalent to showing that

$$
\left\|S\left(\psi_{Q}^{2} S^{*} F\right)\right\|_{L_{x_{2}}^{2} L_{t}^{\infty}} \lesssim \lambda^{-1}|Q|^{1 / 2}\|F\|_{L_{x_{2}}^{2} L_{t}^{1}} .
$$

Let $K\left(x_{2}, t ; x^{\prime}{ }_{2}, t^{\prime}\right)$ be the kernel of the operator $S \chi_{Q} S^{*}$, so that

$$
K\left(x_{2}, t ; x_{2}^{\prime}, t^{\prime}\right)=\int e^{2 \pi i \lambda\left(t|x-y|-t^{\prime}\left|x^{\prime}-y\right|\right)} a\left(t, x, t^{\prime}, x^{\prime}, y\right) \psi_{Q}^{2}(y) d y .
$$

\footnotetext{
${ }^{5}$ For a proof of (4), see below.
} 
Some elementary geometry shows that the phase function $\phi\left(t, x, t^{\prime}, x^{\prime}, y\right)=$ $t|x-y|-t^{\prime}\left|x^{\prime}-y\right|$ is smooth and satisfies

$$
\left|\nabla_{y} \phi\right| \gtrsim\left|x_{2}-x_{2}^{\prime}\right|
$$

uniformly on the support of $t, x, t^{\prime}, x^{\prime}, y$. Thus by the standard non-stationary phase estimate and the properties of $\psi_{B}^{2}(y)$ we have

$$
\left|K\left(x_{2}, t ; x^{\prime}{ }_{2}, t^{\prime}\right)\right| \leq C_{N}|Q|\left(1+\lambda|Q|^{1 / 2}\left|x_{2}-x^{\prime}{ }_{2}\right|\right)^{-N}
$$

for any $N>0$, and (5) follows from Schur's test.

To proceed further it will be convenient to microlocalize the operator in (3), following the ideas of Bourgain [4. This procedure may be viewed as the oscillatory integral-theoretic equivalent of the differentiation operation $\phi \mapsto D \phi$, which lifts a function from a manifold $M$ to its tangent bundle $T M$. Alternatively, one may think of it as a continuous decomposition of the domain and target space into $\lambda^{-1 / 2}$ squares. On a practical level, this microlocalization will isolate certain linearized features of the operator, which can then be handled by standard estimates such as the $L^{4}$ adjoint restriction theorem.

We now turn to the details. To prove (3) we have to show that (suppressing epsilons)

$$
\left\|\int e^{2 \pi i \lambda t|x-y|} a(x, y, t) f(y) d y\right\|_{L_{x}^{q} L_{t}^{\infty}} \lesssim \lambda^{-\frac{1}{2}+\delta}\|f\|_{L_{y}^{p}} .
$$

By introducing two dummy variables $u, v$ supported in $|u|,|v| \lesssim 1$, this is clearly equivalent to the estimate

$$
\left\|\int e^{2 \pi i \lambda t|x-y|} a(x, y, t) a(u) a(v) F(y, v) d y\right\|_{L_{u}^{q} L_{x}^{q} L_{t}^{\infty}} \lesssim \lambda^{-\frac{1}{2}+\delta}\|F\|_{L_{v}^{p} L_{y}^{p}} .
$$

By making the change of variables $x^{\prime}=x+\lambda^{-1 / 2} u, y^{\prime}=y+\lambda^{-1 / 2} v$, this estimate becomes (replacing $x^{\prime}, y^{\prime}$ with $x, y$ )

$$
\begin{aligned}
\| \int e^{2 \pi i \lambda t\left|x+\lambda^{-1 / 2} u-y-\lambda^{-1 / 2} v\right|} a\left(x+\lambda^{-1 / 2} u, y+\lambda^{-1 / 2} v, t\right) & \\
\times & a(u) a(v) F(y, v) d y\left\|_{L_{u}^{q} L_{x}^{q} L_{t}^{\infty}} \lesssim \lambda^{-\frac{1}{2}+\delta}\right\| F \|_{L_{v}^{p} L_{y}^{p} .}
\end{aligned}
$$

However, we have the Taylor expansion

$$
\lambda t\left|x+\lambda^{-1 / 2} u-y-\lambda^{-1 / 2} v\right|=\lambda t r+\lambda^{1 / 2} t u \cdot \omega-\lambda^{1 / 2} t v \cdot \omega+O(1),
$$

where $r=r(x, y), \omega=\omega(x, y)$ are given by

$$
r(x, y)=|x-y|, \quad \omega(x, y)=\frac{x-y}{|x-y|},
$$

and $O(1)$ is a uniformly smooth function of $x, y, t, u, v, \frac{1}{\lambda}$. Thus, by the stability lemma (see Appendices) we see that the above estimate is equivalent to

$$
\|T F\|_{L_{u}^{q} L_{x}^{q} L_{t}^{\infty}} \lesssim \lambda^{-\frac{1}{2}+\delta}\|F\|_{L_{v}^{p} L_{y}^{p}}
$$

where

(7) $T F(x, u, t)=\iint e^{2 \pi i \lambda t r} e^{2 \pi i \lambda^{1 / 2} t u \cdot \omega} e^{-2 \pi i \lambda^{1 / 2} t v \cdot \omega} a(x, y, t, u, v) F(y, v) d y d v$.

It suffices to verify the restricted weak-type estimate

$$
\left|\left\{(x, u): \sup _{t}\left|T \chi_{E}(x, u, t)\right| \gtrsim \alpha\right\}\right| \lesssim \alpha^{-q} \lambda^{\left(-\frac{1}{2}+\delta\right) q}|E|^{q / p}
$$


for arbitrary sets $E \subset\{(y, v):|y|,|v| \lesssim 1\}$ and arbitrary $\alpha>0$, since the original estimate can be recovered (losing an epsilon) by Marcinkiewicz interpolation with the classical estimates. We may assume that $|E|, \alpha$ have polynomial size since (8) follows from classical estimates otherwise. Henceforth $E$ and $\alpha$ are considered fixed.

By microlocalizing the estimate in Proposition 2.1 as above, we obtain our first estimate on $T$ :

Corollary 2.2. If the $y$-support of $F$ is contained in a square $Q$, then

$$
\|T F\|_{L_{u}^{2} L_{x}^{2} L_{t}^{\infty}} \lesssim \lambda^{-1 / 2}|Q|^{1 / 4}\|F\|_{L_{v}^{2} L_{y}^{2}} .
$$

To take advantage of this estimate we perform a variant of the Calderón-Zygmund decomposition. Let $\beta_{1}>0$ be a parameter to be chosen later, and set $E_{Q}=$ $\{(y, v) \in E: y \in Q\}$ for each dyadic square $Q$. Let $\mathcal{Q}$ be a maximal family of disjoint dyadic squares such that

$$
\left|E_{Q}\right| \geq \beta_{1}|Q|^{1 / 2}
$$

for all $Q \in \mathcal{Q}$. We partition $T f$ as

$$
T f=\sum_{Q \in \mathcal{Q}} T \chi_{E_{Q}}+T \chi_{\tilde{E}}
$$

where the "sparse" set $\tilde{E}=E \backslash \bigcup_{Q \in \mathcal{Q}} E_{Q}$ satisfies

$$
|\tilde{E}| \leq|E|, \quad\left|\tilde{E}_{Q}\right|<\beta_{1}|Q|^{1 / 2}
$$

for all squares $Q$. Informally, $\beta_{1}$ represents a measure of the critical "thickness" of the set $E$.

From Proposition 2.2 and (9) we have

$$
\left\|T \chi_{E_{Q}}\right\|_{L_{z}^{2} L_{x}^{2} L_{t}^{\infty}} \lesssim \lambda^{-1 / 2}|Q|^{1 / 4}\left|E_{Q}\right|^{1 / 2} \leq \lambda^{-1 / 2} \beta_{1}^{-1 / 2}\left|E_{Q}\right| .
$$

From the triangle inequality we thus have

$$
\left\|\sum_{Q \in \mathcal{Q}} T \chi_{E_{Q}}\right\|_{L_{z}^{2} L_{x}^{2} L_{t}^{\infty}} \lesssim \lambda^{-1 / 2} \beta_{1}^{-1 / 2}|E| .
$$

From Tchebyshev's inequality we thus have

$$
\left|\left\{(x, u): \sup _{t}\left|\sum_{Q \in \mathcal{Q}} T \chi_{E_{\mathcal{Q}}}(x, u, t)\right| \gtrsim \alpha\right\}\right| \lesssim \lambda^{-1} \beta_{1}^{-1} \alpha^{-2}|E|^{2} .
$$

It remains to estimate the quantity $T \chi_{\tilde{E}}$. From (7) and the change of variables $x-r \omega=y, v=v$ we have

$$
\begin{aligned}
T \chi_{\tilde{E}}(x, u, t)= & \iiint e^{2 \pi i \lambda t r} e^{2 \pi i \lambda^{1 / 2} t u \cdot \omega} e^{-2 \pi i \lambda^{1 / 2} t v \cdot \omega} \\
& \times a(x, r, \omega, t, u, v) \chi_{\tilde{E}}(x-r \omega, v) d r d \omega d v .
\end{aligned}
$$

By a prescient choice of amplitude function, we may assume that

$$
a(x, r, \omega, t, u, v)=a(x) a(r) a(\omega) a(t) a(u) a(v) .
$$

Although the expression (12) appears to be quite complicated, it contains several recognizable parts: a polar coordinates transformation $y=x-r \omega$, a Fourier restriction operator to a circle, a Fourier transform in the $r$ variable, and a Fourier extension operator from a circle. Each individual piece is fairly well-understood, but it is not clear how to coordinate the estimates for the various components. Our 
techniques here are somewhat crude and probably not optimal, but they do give an improvement over the classical results.

Let $\beta_{2}>0$ be a parameter to be chosen later. For each $x$, we associate a set $\Omega_{x}$ of "bad" directions, defined as

$$
\Omega_{x}=\left\{\omega \in S^{1}: \iint \chi_{\tilde{E}}(x-r \omega, v) d r d v>\beta_{2}\right\} .
$$

We thus split $T F=I+I I$, where

$$
\begin{aligned}
I(x, u, t)= & a(x) a(u) a(t) \int_{S^{1} \backslash \Omega_{x}} a(\omega) e^{2 \pi i \lambda^{1 / 2} t u \cdot \omega} \\
& \times\left(\int e^{2 \pi i \lambda t r} e^{-2 \pi i \lambda^{1 / 2} t v \cdot \omega} a(r) a(v) \chi_{\tilde{E}}(x-r \omega, v) d r d v\right) d \omega . \\
I I(x, u, t)= & a(x) a(u) a(t) \int_{\Omega_{x}} a(\omega) e^{2 \pi i \lambda^{1 / 2} t u \cdot \omega} \\
& \times\left(\int e^{2 \pi i \lambda t r} e^{-2 \pi i \lambda^{1 / 2} t v \cdot \omega} a(r) a(v) \chi_{\tilde{E}}(x-r \omega, v) d r d v\right) d \omega .
\end{aligned}
$$

As with $\beta_{1}$, the quantity $\beta_{2}$ is also a measure of the critical thickness of $E$.

To estimate the "good" part $I$, we begin with some $L^{4}$ theory. We first observe that the $t$-Fourier transform of $I$ is essentially supported in the interval $\{\tau:|\tau| \lesssim \lambda\}$. Thus, by Sobolev embedding (or Littlewood-Paley theory) we essentially have

$$
\|I(x, u, \cdot)\|_{L_{t}^{\infty}} \lesssim \lambda^{1 / 4}\|I(x, u, \cdot)\|_{L_{t}^{4}} .
$$

Taking $L_{x}^{4} L_{u}^{4}$ norms of both sides and interchanging some norms we obtain

$$
\|I\|_{L_{x}^{4} L_{u}^{4} L_{t}^{\infty}} \lesssim \lambda^{1 / 4}\|I\|_{L_{x}^{4} L_{t}^{4} L_{u}^{4}} .
$$

To estimate this, we will use the $L^{4}$ adjoint restriction theorem of Fefferman and Stein [12] (see also [9]):

$$
\|f \hat{d} \sigma\|_{L^{4}(B(0, R))} \lesssim R^{\varepsilon}\|f\|_{4} .
$$

Here $d \sigma$ denotes surface measure on $S^{1}$. Applying this to $R=\lambda^{1 / 2} t$ and rescaling, one obtains

$$
\left\|a(u) \int_{S^{1}} f(\omega) e^{2 \pi i \lambda^{1 / 2} t u \cdot \omega} d \omega\right\|_{L_{u}^{4}} \lesssim \lambda^{-1 / 4}\|f\|_{4}
$$

for all $f \in L^{4}\left(S^{1}\right)$, where we have discarded the epsilon factor. Inserting this back into (16), we obtain

$$
\begin{aligned}
\|I\|_{L_{x}^{4} L_{u}^{4} L_{t}^{\infty}}^{4} \lesssim \int & \iint_{S^{1} \backslash \Omega_{x}} a(x) a(t) a(\omega) \\
& \times\left|\int e^{2 \pi i \lambda t r} e^{-2 \pi i \lambda^{1 / 2} t v \cdot \omega} a(r) a(v) \chi_{\tilde{E}}(x-r \omega, v) d r d v\right|^{4} d \omega d t d x .
\end{aligned}
$$

The next step will be to use some $L^{2}$ theory. Since the term inside the absolute values may be crudely estimated by $\beta_{2}$ by (13), we may replace the above estimate 
with

$$
\begin{aligned}
\|I\|_{L_{x}^{4} L_{u}^{4} L_{t}^{\infty}}^{4} \lesssim & \beta_{2}^{2} \iiint a(x) a(t) a(\omega) \\
& \times\left|\int e^{2 \pi i \lambda t r} e^{-2 \pi i \lambda^{1 / 2} t v \cdot \omega} a(r) a(v) \chi_{\tilde{E}}(x-r \omega, v) d r d v\right|^{2} d \omega d t d x \\
= & \beta_{2}^{2} \iint a(x) a(\omega) G(x, \omega) d x d \omega
\end{aligned}
$$

where

$$
G(x, \omega)=\left\|a(t) \int a(r) e^{2 \pi i \lambda t r}\left[\int e^{-2 \pi i \lambda^{1 / 2} t v \cdot \omega} a(v) \chi_{\tilde{E}}(x-r \omega, v) d v\right] d r\right\|_{L_{t}^{2}}^{2} .
$$

For each $r$, the term in brackets has a $t$-Fourier transform essentially supported in the interval $\left\{\tau:|\tau-\lambda r| \lesssim \lambda^{1 / 2} \mid\right\}$. Thus, the contribution from $r=r^{1}$ and $r=r^{2}$ will be orthogonal whenever $\left|r^{1}-r^{2}\right| \gg \lambda^{-1 / 2}$. Thus, by the usual orthogonality arguments we have

$$
G(x, \omega) \lesssim \lambda^{-1 / 2} \int a(r)\left\|\int e^{-2 \pi i \lambda^{1 / 2} t v \cdot \omega} a(v) \chi_{\tilde{E}}(x-r \omega, v) d v\right\|_{L_{t}^{2}}^{2} d r .
$$

Inserting this back into (18), we obtain

$$
\begin{aligned}
\|I\|_{L_{x}^{4} L_{u}^{4} L_{t}^{\infty}} \lesssim & \lambda^{-1 / 2} \beta_{2}^{2} \iiint \int a(x) a(\omega) a(r) a(t) \\
& \times\left|\int e^{-2 \pi i \lambda^{1 / 2} t v \cdot \omega} a(v) \chi_{\tilde{E}}(x-r \omega, v) d v\right|^{2} d t d r d \omega d x .
\end{aligned}
$$

Making the change of variables $y=x-r \omega, t=t, r=r, \omega=\omega$ and adjusting the amplitude functions, we obtain

$$
\begin{aligned}
\|I\|_{L_{x}^{4} L_{u}^{4} L_{t}^{\infty}} \lesssim & \lambda^{-1 / 2} \beta_{2}^{2} \iiint \int a(y) a(\omega) a(r) a(t) \\
& \times\left|\int e^{-2 \pi i \lambda^{1 / 2} t v \cdot \omega} a(v) \chi_{\tilde{E}}(y, v) d v\right|^{2} d \omega d t d r d y \\
= & \lambda^{-1 / 2} \beta_{2}^{2} \iiint \int a(y) a(\omega) a(r) a(t)\left|H\left(y, \lambda^{1 / 2} t \omega\right)\right|^{2} d \omega d t d r d y
\end{aligned}
$$

where

$$
H(y, \eta)=\int e^{-2 \pi i v \cdot \eta} a(v) \chi_{\tilde{E}}(y, v) d v
$$

is the $v$-Fourier transform of $a(v) \chi_{\tilde{E}}(y, v)$. The $r$ integration is now trivial and can be discarded. Applying the change of variables $\eta=\lambda^{1 / 2} t \omega, y=y$ we see that (19) becomes

$$
\|I\|_{L_{x}^{4} L_{u}^{4} L_{t}^{\infty}}^{4} \lesssim \lambda^{-1 / 2} \beta_{2}^{2} \int a(y) \lambda^{-1}\|H(y, \cdot)\|_{L_{\eta}^{2}}^{2} d y .
$$

However, by Plancherel's theorem we have

$$
\|H(y, \cdot)\|_{L_{\eta}^{2}}^{2}=\left\|a(\cdot) \chi_{\tilde{E}}(y, \cdot)\right\|_{L_{v}^{2}}^{2} \lesssim \int \chi_{\tilde{E}}(y, v) d v
$$


and so

$$
\|I\|_{L_{x}^{4} L_{u}^{4} L_{t}^{\infty}} \lesssim \lambda^{-3 / 2} \beta_{2}^{2}|E| .
$$

Applying Tchebyshev's inequality we obtain

$$
\left\{(x, u):\left|\sup _{t} I(x, u, t)\right| \gtrsim \alpha\right\} \lesssim \alpha^{-4} \lambda^{-3 / 2} \beta_{2}^{2}|E| .
$$

We now turn to the quantity $I I(x, u, t)$ in (15), using a variant of an argument of Cordoba. We use crude estimates and (13) to obtain the estimate

$$
\begin{aligned}
\left|\sup _{t} I I(x, u, t)\right| \lesssim a(x) a(u) \int_{\Omega_{x}} \iint \chi_{\tilde{E}}(x-r \omega, v) d r d v d \omega \\
\quad \lesssim a(x) a(u) \int \beta_{2}^{-1}\left(\iint \chi_{\tilde{E}}(x-r \omega, v) d r d v\right)^{2} d \omega \\
\quad=a(x) a(u) \beta_{2}^{-1} \iiint \iint \chi_{\tilde{E}}(x-r \omega, v) \chi_{\tilde{E}}\left(x-r^{\prime} \omega, v^{\prime}\right) d r d r^{\prime} d v d v^{\prime} d \omega .
\end{aligned}
$$

Integrating this in $x$ and $u$ we obtain

$$
\begin{aligned}
& \iint\left|\sup _{t} I I(x, u, t)\right| d x d u \\
& \quad \lesssim \beta_{2}^{-1} \iiint \iiint \chi_{\tilde{E}}(x-r \omega, v) \chi_{\tilde{E}}\left(x-r^{\prime} \omega, v^{\prime}\right) d x d r d r^{\prime} d v d v^{\prime} d \omega .
\end{aligned}
$$

By the change of variables $y=x-r \omega, y^{\prime}=y-r^{\prime} \omega, v=v, v^{\prime}=v^{\prime}$ we can rewrite the right-hand side as a capacity integral:

$$
\iint\left|\sup _{t} I I(x, u, t)\right| d x d u \lesssim \beta_{2}^{-1} \iiint \int \chi_{\tilde{E}}(y, v) \chi_{\tilde{E}}\left(y^{\prime}, v^{\prime}\right) \frac{d y d y^{\prime} d v d v^{\prime}}{\left|y-y^{\prime}\right|} .
$$

By estimating the $d y^{\prime} d v^{\prime}$ integral by its supremum, we obtain

$$
\iint\left|\sup _{t} I I(x, u, t)\right| d x d u \lesssim \beta_{2}^{-1}|E| \sup _{y} \iint \chi_{\tilde{E}}\left(y^{\prime}, v^{\prime}\right) \frac{d y^{\prime} d v^{\prime}}{\left|y-y^{\prime}\right|} .
$$

However, we may estimate the integral dyadically as

$$
\iint \chi_{\tilde{E}}\left(y^{\prime}, v^{\prime}\right) \frac{d y^{\prime} d v^{\prime}}{\left|y-y^{\prime}\right|} \lesssim \lambda^{-C}+\sum_{k=0}^{C \log \lambda} 2^{k}\left|\left\{\left(y^{\prime}, v^{\prime}\right) \in \tilde{E}:\left|y^{\prime}-y\right| \sim 2^{k}\right\}\right| .
$$

for some large $C>0$. Since (10) implies

$$
\left|\left\{\left(y^{\prime}, v^{\prime}\right) \in \tilde{E}:\left|y^{\prime}-y\right| \sim 2^{k}\right\}\right| \lesssim \beta_{1} 2^{-k},
$$

we thus see that (21) reduces to (ignoring epsilons)

$$
\iint\left|\sup _{t} I I(x, u, t)\right| d x d u \lesssim \beta_{1} \beta_{2}^{-1}|E| .
$$

Thus by Tchebyshev's inequality, we obtain

$$
\left|\left\{(x, u):\left|\sup _{t} I I(x, u, t)\right| \gtrsim \alpha\right\}\right| \lesssim \alpha^{-1} \beta_{1} \beta_{2}^{-1}|E| .
$$

Combining this with (20) and (11) we obtain

$$
\begin{aligned}
\mid\{(x, u) & \left.: \sup _{t}\left|T \chi_{E}(x, u, t)\right| \gtrsim \alpha\right\} \mid \\
& \lesssim \lambda^{-1} \beta_{1}^{-1} \alpha^{-2}|E|^{2}+\alpha^{-4} \lambda^{-3 / 2} \beta_{2}^{2}|E|+\alpha^{-1} \beta_{1} \beta_{2}^{-1}|E| .
\end{aligned}
$$


We optimize in $\beta_{1}$ and $\beta_{2}$, choosing

$$
\beta_{1}=\lambda^{-3 / 10}|E|^{3 / 5}, \quad \beta_{2}=\lambda^{2 / 5} \alpha|E|^{1 / 5},
$$

obtaining

$$
\left|\left\{(x, u): \sup _{t}\left|T \chi_{E}(x, u, t)\right| \gtrsim \alpha\right\}\right| \lesssim \alpha^{-2} \lambda^{-7 / 10}|E|^{7 / 5},
$$

which is (8) for $p=10 / 7, q=2, \delta=3 / 20$, as claimed.

The results are not best possible. One largely unexploited gain lies in the curvature inherent in the $(r, \omega)$ system of coordinates, as $x$ varies; apart from the $L^{4}$ adjoint restriction theorem, curvature is barely utilized in the above arguments. For instance, one could try to apply the adjoint of (17) to control the dependence on the variable $v$. It is likely that one needs estimates that exploit the structure of the operator $T$ as a whole, rather than composing together independent estimates for the various pieces.

A good model case occurs when $|E| \sim \alpha \sim \lambda^{-7 / 6}, \beta_{1} \sim \beta_{2} \sim \lambda^{-1}$, in which case (23) gives only the trivial bound

$$
\left|\left\{(x, u): \sup _{t}\left|T \chi_{E}(x, u, t)\right| \gtrsim \alpha\right\}\right| \lesssim 1 .
$$

Any improvement on (11), (20) or (22) in this case is likely to yield some progress on Theorem [1.2.

Another possibility, pointed out to the author by Chris Sogge, is to try to prove the estimate

$$
\|S f\|_{L_{x}^{4 / 3} L_{t}^{\infty}} \lesssim \lambda^{-3 / 8+\varepsilon}\|f\|_{4 / 3}
$$

for all $\varepsilon>0$; this corresponds to the point $b$ in Figure 1 . The dual of this estimate is

$$
\left\|S^{*} F\right\|_{4} \lesssim \lambda^{-3 / 8+\varepsilon}\|F\|_{L_{x}^{4} L_{t}^{1}}
$$

and it may be possible to show this by computing the $L^{4}$ norm explicitly. Unfortunately the $L_{t}^{1}$ norm on the right-hand side makes this very difficult, as one is quickly faced with the task of estimating an oscillatory integral such as

$$
\left\|\int e^{2 \pi i t(x)|x-y|} F(x) d x\right\|_{L_{y}^{4}}^{4}
$$

for some arbitrary rough function $t(x)$. Although the $y$ integration can more or less be computed explicitly, it is unclear to us how to handle the (very rough) $x$ integrations.

It may be possible to sharpen (24), replacing the norm $\|f\|_{4 / 3}$ by $\|f\|_{2}$, or even freezing the $y_{1}$ variable as in the proof of Proposition 2.1. The Knapp example and the example in (2) suggest that these sharpened estimates are significant.

The quantities $\beta_{1}$ and $\beta_{2}$ both measure how "thick" the set $E$ is. Since these quantities seem to be comparable to $\lambda^{-1}$ in the critical cases, we thus see the qualitative phenomenon that the worst types of sets $E$ are those which are effectively one-dimensional. The example (2) seems to be consistent with this observation. 


\section{Appendix: A StABILITy LEMma FOR OsCILlatory integrals}

In this section we make precise the heuristic that one may modify the phase or amplitude of an oscillatory integral by a smooth $O(1)$ factor without affecting its regularity properties. Versions of this principle have appeared before, see e.g. [26], [1], 14.

Definition 3.1. A Banach space $X$ of functions on a compact subset of $\mathbf{R}^{n}$ is said to be well-behaved if one has

$$
\left\|e^{2 \pi i\langle\cdot \xi\rangle} f\right\|_{X} \lesssim(1+|\xi|)^{M}\|f\|_{X}
$$

for all $f \in X, \xi \in\left(\mathbf{R}^{n}\right)^{*}$ and some large $M>0$.

All the Banach spaces used in this paper are well-behaved. We remark that the results in this Appendix can also be extended to well-behaved quasi-Banach spaces, since the absolute summability of functions with rapidly decreasing quasi-norms can be demonstrated easily.

Lemma 3.2. For each $R \gg 1$, let $K_{R}(x, y)$ be a bounded, compactly supported function on $\boldsymbol{R}^{n} \times \boldsymbol{R}^{m}$, where the bounds and support are independent of $R$, and let $X, Y$ be well-behaved Banach spaces on the $x$ and $y$ supports of $K$ respectively. Suppose $b(x, y, \tau)$ is a bounded function which is $C^{\infty}$ in $x, y$ and $\tau$, for $0 \leq \tau \lesssim 1$ and $x, y$ in the support of $K$. Then, if the operators $A_{R}$ and $B_{R}$ are defined by

$$
\begin{gathered}
A_{R} f(x)=\int K_{R}(x, y) f(y) d y, \\
B_{R} f(x)=\int K_{R}(x, y) b(x, y, 1 / R) f(y) d y,
\end{gathered}
$$

then for all $N>0$ we have

$$
\left\|B_{R}\right\|_{X \rightarrow Y} \lesssim\left\|A_{R}\right\|_{X \rightarrow Y}+O\left(R^{-N}\right)
$$

In particular, if $b=e^{i a}$ for some uniformly smooth $a(x, y, \tau)$ then the operator norms of $A_{R}$ and $B_{R}$ are comparable (except for a rapidly decreasing error). In practice the error can be ignored since there is usually a trivial counterexample which shows that $\left\|A_{R}\right\|_{X \rightarrow Y},\left\|B_{R}\right\|_{X \rightarrow Y}$ is not rapidly decreasing in $R$.

Proof. Since $x, y$ are compactly supported, one can break $b$ up into a Fourier series in $x, y$ and a Taylor series in $\tau$ as

$$
b(x, y, \tau)=\sum_{j=0}^{N} \sum_{k \in \mathbf{Z}^{n}} \sum_{l \in \mathbf{Z}^{m}} c_{j, k, l} \tau^{j} e^{2 \pi i\langle k, x\rangle} e^{2 \pi i\langle l, y\rangle}+O\left(\tau^{N}\right),
$$

where the $f_{j, k}$ are uniformly bounded functions, the $c_{j, k, l}$ are constants which are rapidly decreasing in $k, l$, the sets $\mathbf{Z}^{n}, \mathbf{Z}^{m}$ are suitable lattices, and $N$ is an arbitrarily large number. The result then follows from the operator identity

$$
B_{R}=\sum_{j=0}^{N} \sum_{k \in \mathbf{Z}^{n}} \sum_{l \in \mathbf{Z}^{m}} c_{j, k, l} R^{-j} e^{2 \pi i\langle k, \cdot\rangle} A_{R} e^{2 \pi i\langle l, \cdot\rangle}+O\left(R^{-N}\right),
$$

the assumption of well-behavedness, the rapid decrease of $c_{j, k, l}$, and the triangle inequality. 


\section{Appendix: Reduction to Proposition 1.3}

In this section we fill in a gap in the derivation of Theorem 1.2, namely the implication of (11) from Proposition 1.3. This is an immediate consequence of the following proposition.

Proposition 4.1. 27] Suppose $1<p<2, \delta_{0}>0$ were such that (3) held for $\delta=\delta_{0}$, all $\varepsilon>0$ and all suitable choices of bump function a (with the constants depending on $\varepsilon$ and $a$ ). Then (11) holds for all $\delta>\delta_{0}$, and one has almost everywhere convergence of Bochner-Riesz means of $L^{p}$ functions for this range of $\delta$.

For a full proof of this proposition, see [27], Proposition 5.3; we give only an informal outline here.

It suffices to prove (11). The kernel of $S_{t}^{\delta}$ can be dyadically decomposed in the usual manner into a harmless term supported on $\{|x| \lesssim t\}$ (which can be controlled by the Hardy-Littlewood maximal function), together with a term $K_{t}^{\delta, j}$ supported on $\left\{|x| \sim 2^{j} t\right\}$ for each $j>0$. It suffices to control the contributions of $K_{t}^{\delta, j}$ uniformly in $j$, since one can improve this uniform control to an exponentially decreasing control by worsening $\delta$ slightly.

Fix $j$. Since every $t>0$ is in a union of intervals $J_{l}=\bigcup_{k>0}\left[2^{2 j k+l}, 2^{2 j k+l+1}\right]$ for some $0 \leq l<2 j$, we will give up a factor of $2 j$ (which can be absorbed by worsening $\delta$ slightly) and assume that $t$ is restricted to $J_{l}$ for a fixed $0 \leq l<2 j$.

By the usual Calderón-Zygmund theory adapted to weak $(p, p)$ estimates (see e.g. 12 ), we may replace the function $f$ with a collection of functions $b_{I}$ supported on disjoint dyadic cubes $I$, which are suitably normalized in $L^{p}$. From the CalderónZygmund construction we may assume arbitrarily many moment conditions on the $b_{I}$.

Fix a single $b_{I}$, and suppose that $I$ has sidelength $2^{i}$. We claim that the only significant contribution of $b_{I}$ occurs when $2^{-j} t \lesssim 2^{i} \lesssim 2^{j} t$. Indeed, when $2^{j} t \ll 2^{i}$ the contribution of $b_{I}$ is supported in a constant dilate of $I$, while when $t \gg$ $2^{-j} t \gg 2^{i}$ the kernel $K_{t}^{\delta, j}$ is essentially constant at the scale of $2^{i}$, and one can get an arbitrarily good estimate on $b_{I} * K_{t}^{\delta, j}$ by exploiting the moment conditions on $b_{I}$.

Combining this observation with the restriction $t \in J_{l}$, we see that for each $b_{I}$ there is only a single dyadic interval of $t$ for which the contribution of $b_{I}$ is significant. This allows us to decouple the various dyadic intervals in $J_{l}$ and assume that $t$ is restricted to a single dyadic interval $t \in\left[2^{m}, 2^{m+1}\right]$. Since the kernels $K_{t}^{\delta, j}$ are now supported in a ball $B\left(0, C 2^{j} 2^{m}\right)$, we may restrict the support of $f$ to a ball of radius $C 2^{j} 2^{m}$. By using the standard stationary phase asymptotics for $K_{t}^{\delta, j}$ and rescaling, one is quickly led to an estimate of the form (3), which is given by hypothesis. This finishes the proof.

\section{REFERENCES}

[1] J. Bourgain, Besicovitch-type maximal operators and applications to Fourier analysis, Geom. and Funct. Anal. 22 (1991), 147-187. MR 92g:42010

[2] J. Bourgain, A remark on Schrodinger operators, Israel J. Math. 77 (1992), 1-16. MR 93k:35071

[3] J. Bourgain, Estimates for cone multipliers, Operator Theory: Advances and Applications, 77 (1995), 41-60. MR 96m:42022

[4] J. Bourgain, Some new estimates on oscillatory integrals, Essays in Fourier Analysis in Honor of E. M. Stein, Princeton University Press (1995), 83-112. MR 96c:42028 
[5] J. Bourgain, Refinements of Strichartz' Inequality and Applications to 2D-NLS with Critical Nonlinearity, Internat. Math. Res. Notes 1998, no. 5, 253-283. MR 99f:35184

[6] A. Carbery, The boundedness of the maximal Bochner-Riesz operator on $L^{4}\left(\boldsymbol{R}^{2}\right)$, Duke Math. J. 50 (1983), 409-416. MR 84m:42025

[7] A. Carbery, Variants of the Caldersn-Zygmund theory for $L^{p}$-spaces, Rev. Mat. Iberoamericana 2 (1986), 381-396. MR 89f:42011

[8] A. Carbery; J. L. Rubio de Francia, L. Vega, Almost everywhere summability of Fourier integrals. J. London Math. Soc. 38 (1988), 513-524. MR 90e:42033

[9] L. Carleson and P. Sjölin, Oscillatory integrals and a multiplier problem for the disc, Studia Math. 44 (1972): 287-299. MR 50:14052

[10] L. Chen, D. Fan, The convergence of the Bochner-Riesz means at the critical index, Proc. Amer. Math. Soc. 124 (1996). MR 96k:42014

[11] M. Christ, On the regularity of inverses of singular integral operators, Duke Math. J., 57 (1988): 459-484. MR 90c:42022

[12] C. Fefferman, Inequalities for strongly singular convolution operators, Acta Math. 124 (1970), 9-36. MR 41:2468

[13] C. Fefferman, The multiplier problem for the ball, Ann. of Math. 94 (1971): 330-336. MR 45:5661

[14] I. L. Hwang, The $L^{2}$-boundedness of pseudodifferential operators, Trans. Amer. Math. Soc. 302 (1987), 55-76. MR 88e:47096

[15] Y. Kanjin, Convergence almost everywhere of Bochner-Riesz means for radial functions, Ann. Sci. Kanazawa Univ. 25 (1988): 11-15. MR 90b:42034

[16] M. Kojima, On the almost everywhere convergence of Bochner-Riesz means of multiple Fourier integrals for radial functions., Nihonkai Math. J. 3 (1992), 9-21. MR 93f:42027

[17] S. Z. Lu, Decomposition of kernel and maximal generalized Bochner-Riesz means, Chinese Quart. J. Math. 4 (1989): 16-23. MR 90k:42016

[18] A. Moyua, A. Vargas, L. Vega, Schrödinger Maximal Function and Restriction Properties of the Fourier Transform, International Math. Research Notices 1996, no. 16, 793-815. MR 97k:42042

[19] A. Moyua, A. Vargas, L. Vega, Restriction theorems and maximal operators related to oscillatory integrals in $\boldsymbol{R}^{3}$, Duke Math. J. 96 (1999), 547-574. MR 2000b:42017

[20] E. M. Stein, Interpolation of linear operators, Trans. Amer. Math. Soc. 83 (1956): 482-492. MR 18:575d

[21] E. M. Stein, On limits of sequences of operators, Ann. of Math. 74 (1961): 140-170. MR 23:A2695

[22] E. M. Stein, Some problems in harmonic analysis, Harmonic analysis in Euclidean spaces (Proc. Sympos. Pure Math., Williams Coll., Williamstown, Mass., 1978), Part 1, pp. 3-20. MR 80m:42027

[23] E. M. Stein, G. Weiss, Introduction to Fourier Analysis on Euclidean Spaces, Princeton University Press, 1971. MR 46:4102

[24] E. M. Stein, Harmonic Analysis, Princeton University Press, 1993. MR 95c:42002

[25] E. M. Stein, M. H. Taibleson, and G. Weiss, Weak-type estimates for maximal operators on certain $H^{p}$ spaces, Rend. Circ. Mat. Palermo suppl. 1 (1981): 81-97. MR 83c:42017

[26] T. Tao, The Bochner-Riesz conjecture implies the restriction conjecture, Duke Math J. 96 (1999), 363-375. MR 2000a:42023

[27] T. Tao, The weak-type endpoint Bochner-Riesz conjecture and related topics, Indiana Univ. Math. J. 47 (1998), 1097-1124. MR 2000a:42024

[28] T. Tao, A. Vargas, L. Vega, A bilinear approach to the restriction and Kakeya conjectures, J. Amer. Math. Soc. 11 (1998), 967-1000. MR 99f:42026

Department of Mathematics, University of California, los Angeles, Los Angeles, CALIFORNia 90024

E-mail address: tao@math.ucla.edu 